\title{
Stereodivergent Asymmetric Reactions Catalyzed by Brucine Diol
}

\author{
Hun Young Kim \\ Kyungsoo Oh* \\ Synlett 2015, 26, DOI: 10.1055/s-0034-1380862.
}

The advance online publication (e-First) version of this article contained errors in the Evans model presented in Figure 4. Further, the Acknowledgment section has been corrected for both the current online version and the print publication. The editorial office apologizes profusely for these mistakes. 